\title{
Cerenkov radiation of longitudinal photons by neutrinos
}

\author{
Sarira Sahu ${ }^{1}$ \\ Theory Group, Physical Research Laboratory, \\ Navrangpura, Ahmedabad-380 009 \\ India
}

\begin{abstract}
In a relativistic plasma neutrino can emit plasmons by the Cerenkov process which is kinematically allowed for a range of frequencies for which refractive index is greater than one. We have calculated the rate of energy emission by this process. We compute the energy deposited in a stalled supernova shock wave by the Cerenkov process and find that it is much smaller than the Bethe-Wilson mechanism.
\end{abstract}

${ }^{1}$ email:sarira@prl.ernet.in 


\section{Introduction}

The electromagnetic wave passing throuth the plasma, is modified because of the mobile charged particle in the medium and it consists of coherent vibration of the electromagnetic field as well as the density of the charged particles[1]. Photons no longer propagate at the speed of light and satisfy the dispersion relations for transverse and longitudinal modes. Transverse photons are similar to the ordinary photons in the vacuum and longitudinal photons are the collective excitation of the plasma known as "plasmon". Cerenkov radiation is emitted when a charge particle moves through a medium with a velocity greater than $c / n, n$ being the refractive index of the medium. This is also true for neutral particles with non-zero magnetic and or electric dipole moments. For Cerenkov radiation to take place in the medium, the refractive index of photon should satisfy the condition $n=$ $|\mathbf{k}| / w>1$, where $\mathbf{k}$ and $\omega$ are momentum and frequency of the emitted photon respectively. Recently several authors have considered the Cerenkov radiation emitted by neutrinos as they pass through a medium[2, 3, 4, 5, 6]. Olivo, Nieves and Pal have recently shown that, neutrino can emit Cerenkov radiation even in the massless limit and having no electromagnetic dipole moments[2].

In a relativistic plasma neutrinos can loose energy by the Cerenkov radiation of plasmon. This takes place: firstly because neutrinos acquire an effective charge in the medium, by coupling to the electromagnetic field through electrons and positrons in the plasma[2, 3, 7], which is shown in the Feynman diagrams in figure 1. Secondly there is a range of frequencies of plasmon for which the refractive index $n>1$, and the Cerenkov process is kinematically allowed. We compute the rate of energy radiated by neutrinos (even those with zero electromagnetic dipole moments) in relativistic plasma. We find that the rate of energy radiated in the form of plasmon is

$$
S \simeq \frac{T}{8 \pi^{2} \alpha} G_{F}^{2} C_{V}^{2} P_{l}^{2}(n-1)^{3} E_{1}^{2}
$$

where $E_{1}$ is the incoming neutrino energy. We calculate the energy deposition 
by this process in the stalled shock wave of the supernova and compare with the Bethe-Wilson (BW) mechanism of shock revival. We found that the Cerenkov process is very weak compared to the $\mathrm{BW}$ mechanism.

\section{Cerenkov process}

The dispersion relations satisfy by the transverse and longitudinal modes of photon depend on the properties of the plasma. In the relativistic limit the dispersion relations are given by [8]

$$
w_{t}^{2}=k^{2}+w_{p}^{2} \frac{3 w_{t}^{2}}{2 k^{2}}\left(1-\frac{\left(w_{t}^{2}-k^{2}\right) w_{t}}{w_{t}^{2} 2 k} \log \left|\frac{w_{t}+k}{w_{t}-k}\right|\right) \quad 0 \leq k<\infty
$$

and

$$
w_{l}^{2}=w_{p}^{2} \frac{3 w_{l}^{2}}{k^{2}}\left(\frac{w_{l}}{2 k} \log \left|\frac{w_{l}+k}{w_{l}-k}\right|-1\right) 0 \leq k<\infty .
$$

for the transverse and longitudinal modes respectively. In the above equations $w_{p}$ is the plasma frequency and $k$ is the photon momentum. In the medium with refractive index $n_{\alpha}=|k| / w_{\alpha}$, the above dispersion relations can be expressed as

$$
\left(\frac{w_{t}}{w_{p}}\right)^{2}=\frac{3}{2 n_{t}^{2}\left(1-n_{t}^{2}\right)}\left(1-\frac{\left(1-n_{t}^{2}\right)}{2 n_{t}} \log \left|\frac{1+n_{t}}{1-n_{t}}\right|\right)
$$

and

$$
\left(\frac{w_{l}}{w_{p}}\right)^{2}=\frac{3}{n_{l}^{3}}\left(\frac{1}{2} \log \left|\frac{1+n_{l}}{1-n_{l}}\right|-n_{l}\right) .
$$

For $n>1,\left(w_{t} / w_{p}\right)^{2}$ is always negative but for a range of $n>1 ;\left(w_{l} / w_{p}\right)^{2}$ is positive which is shown in figure 1. From the dispersion relations we see that the refractive index $n_{t}=w_{t} /|k|$ of transverse photon is always less than one, so transverse photon can not be emitted by Cerenkov process in a plasma. On the other hand, for longitudinal photon we see that $n_{l}$ can be greater than one. So there can be plasmon emission by Cerenkov process. Recently several authors have considered the Cerenkov radiation by neutrinos in a medium[2, †]. Neutrino

properties get modified when propagates through a medium as a consequence of the weak interaction with the background particles [7]. It has been shown earlier 
Fig. 1: RHS of eq.(4) is ploted as a function of $n_{l}$.

that neutrino acquires an effective charge in the medium by coupling to the electromagnetic field through electrons and positrons in the plasma[2, 3].

Here we will consider the Cerenkov process

$$
\nu\left(p_{1}\right) \rightarrow \nu\left(p_{2}\right)+\gamma(k)
$$

in the medium, where $\gamma(k)$ is the plasmon (longitudinal photon) emitted with a momentum k. Feynman diagram for the above processes are shown in figure 2. 
Fig. 2: Feynman diagram for neutrino photon coupling through $W$ and $Z$ exchange in the medium.

The matrix element for the above process is given by

$$
\mathcal{M}=\frac{G_{F}}{\sqrt{2}} \Gamma^{\alpha \mu} \epsilon_{\mu}(\mathbf{k}, \lambda) \bar{u}\left(p_{2}\right) \gamma_{\alpha}\left(1-\gamma_{5}\right) u\left(p_{1}\right)
$$

where $\Gamma^{\alpha \mu}(w, \mathbf{k})$ is the effective vertex for the plasmon interac ing with the neutrino current. This effective vertex is due to the $\mathrm{W}$ and $\mathrm{Z}$ in the loops in the Feynman diagram [3, 8]. The vertex tensor is gauge invariant quantity, as $k_{\mu} \Gamma^{\alpha \mu}=$ $0[3,8]$. The effective vertex tensor is [8]

$$
\begin{aligned}
\Gamma^{\alpha \mu}(w, \mathbf{k}) & =\frac{1}{\sqrt{4 \pi \alpha}}\left(C_{V} P_{l}\left(1, \frac{w}{k} \hat{k}\right)^{\alpha}\left(1, \frac{w}{k} \hat{k}\right)^{\mu}\right. \\
& \left.+g^{\alpha i}\left[C_{V} P_{t}\left(\delta^{i j}-\hat{k}^{i} \hat{k}^{j}\right)+C_{A} \Pi_{A}\left(i \epsilon^{i j k}\right)\right] g^{j \mu}\right)
\end{aligned}
$$

where $C_{V}$ and $C_{A}$ are vector and axial vector coefficients, $\epsilon_{\mu}(k, \lambda)$ is the polarization vector, $u\left(p_{i}\right)$ is the neutrino spinor and $\alpha$ in the denominator is the electromagnetic coupling constant. The functions $P_{l}, P_{t}$ and $\Pi_{A}$ are longitudinal, transverse and axial polarization functions respectively[ [8]. As we have already shown, the longitudinal part will only contribute to the above process, so transverse and axial parts (second and third terms in eq.(7)) of the vertex are ignored. The longitudinal polarization function for plasmon is given by [8]

$$
P_{l}=3 w_{p}^{2}\left(\frac{1}{2 n_{l}} \log \left|\frac{1+n_{l}}{1-n_{l}}\right|-1\right),
$$


where $w_{p}^{2}=4 \pi e^{2} N_{e} / m_{e}$ is the plasma frequency. From eq.(6), $|\mathcal{M}|^{2}$ is

$$
|\mathcal{M}|^{2}=\frac{G_{F}^{2}}{2} \sum_{\lambda} \epsilon_{\mu}(\mathbf{k}, \lambda) \epsilon_{\delta}^{*}(\mathbf{k}, \lambda) \Gamma^{\alpha \mu} \Gamma^{* \beta \delta} 8\left(p_{2 \alpha} p_{1 \beta}-\left(p_{1} . p_{2}\right) g_{\alpha \beta}+p_{2 \beta} p_{1 \alpha}\right) .
$$

Henceforth we will be using $n_{l}=n$ and $w_{l}=w$. Using the polarization sum in the medium

$$
\sum_{\lambda} \epsilon_{\mu}(\mathbf{k}, \lambda) \epsilon_{\delta}^{*}(\mathbf{k}, \lambda)=-g_{\mu \delta}+\left(1-\frac{1}{n^{2}}\right) W_{\mu} W_{\delta}+\frac{1}{n^{2} w}\left(W_{\mu} k_{\delta}+k_{\mu} W_{\delta}\right)-\frac{1}{n^{2} w^{2}} k_{\mu} k_{\delta}
$$

with $W_{\mu}=(1,0)$ the center of mass velocity of the medium. Putting this in eq.(9) we obtain for the longitudinal part,

$$
\begin{aligned}
|\mathcal{M}|^{2}= & \frac{G_{F}^{2}}{2} C_{V}^{2} P_{l}^{2} \frac{1}{4 \pi \alpha} 8\left(1-\frac{1}{n^{2}}\right)\left[2\left(E_{2}-\left(\mathbf{p}_{\mathbf{2}} \cdot \mathbf{k}\right) \frac{w}{k^{2}}\right)\right. \\
& \left(E_{1}-\left(\mathbf{p}_{\mathbf{1}} \cdot \mathbf{k}\right) \frac{w}{k^{2}}\right)-\left(E_{1} E_{2}-\left(\mathbf{p}_{\mathbf{1}} \cdot \mathbf{p}_{\mathbf{2}}\right)\left(1-\frac{w^{2}}{k^{2}}\right)\right]
\end{aligned}
$$

where $p_{1}=\left(E_{1}, \mathbf{p}_{1}\right), p_{2}=\left(E_{2}, \mathbf{p}_{\mathbf{2}}\right)$ and $k=(w, \mathbf{k})$ are the four-momenta of incoming neutrino, outgoing neutrino and outgoing photon respectively. Then the total energy emitted from a single process is

$$
S=\frac{1}{2 E_{1}} \int \frac{d^{3} p_{2}}{2 E_{2}(2 \pi)^{3}} \frac{d^{3} k}{2 w(2 \pi)^{3}} w(2 \pi)^{4} \delta^{4}\left(p_{1}-p_{2}-k\right)|\mathcal{M}|^{2}
$$

Using the identity

$$
\int \frac{d^{3} p_{2}}{2 E_{2}}=\int d^{4} p_{2} \Theta\left(E_{2}\right) \delta\left(p_{2}^{2}-m_{\nu}^{2}\right)
$$

where $\Theta\left(E_{2}\right)$ is the step function and $m_{\nu}$ is the neutrino mass. Putting eq.(13) in eq.(12) and integrating over $p_{2}$ we obtain for energy radiated by neutrino in time $\mathrm{T}$ is

$$
S=\frac{T}{16 \pi^{2} E_{1}} \int \frac{d^{3} k}{2\left|\mathbf{p}_{\mathbf{1}}\right||\mathbf{k}|} \delta\left(\frac{\left(2 E_{1} w-w^{2}+k^{2}\right)}{2\left|\mathbf{p}_{\mathbf{1}}\right||\mathbf{k}|}-\cos \theta\right)|\mathcal{M}|^{2} .
$$

The angle $\theta$ between the incoming neutrino and the emitted plasmon is obtained from the delta function in eq.(14),

$$
\cos \theta=\frac{\left(2 E_{1} w-w^{2}+k^{2}\right)}{2\left|\mathbf{p}_{\mathbf{1}}\right||\mathbf{k}|}=\frac{1}{n v}\left(1+\frac{\left(n^{2}-1\right) w}{2 E_{1}}\right),
$$


where $v=\frac{\left|\mathbf{p}_{1}\right|}{E_{1}}$ is the neutrino velocity $(\simeq 1)$. Since $-1 \leq \cos \theta \leq 1$; which implies

$$
-\frac{2 E_{1}}{(n-1)} \leq w \leq \frac{2 E_{1}}{(n+1)}
$$

But definitely $-\frac{2 E_{1}}{(n-1)}$ can not be the lower limit for the above Cerenkov process, as for $n>1$ this is a negative quantity and $w$ can not be negative. On the other hand from the dispersion relation for the longitudinal photon we obtain $w \geq 0.035 w_{p}$. Thus the kinematically allowed region for the Cerenkov process is

$$
0.35 w_{p} \leq w \leq \frac{2 E_{1}}{(n+1)}
$$

Evaluating $|\mathcal{M}|^{2}$ and simplifying the eq.(14) we obtain

$$
S=\frac{T}{8 \pi^{2} \alpha} G_{F}^{2} C_{V}^{2} \int_{w_{1}}^{w_{2}} \frac{\left(n^{2}-1\right)^{3}}{n^{4}} w\left(1-\frac{w}{E_{1}}\right) P_{l}^{2} d w
$$

where $C_{V}=\left(2 \sin ^{2} \theta_{W} \pm \frac{1}{2}\right)$ for $\nu_{e}, \nu_{\mu}$ and $w_{1}$ and $w_{2}$ are the lower and upper limits of $w, \theta_{W}$ is the weak mixing angle and $\sin ^{2} \theta_{W} \simeq 0.233$. As the plasmon emission is possible for a narrow range of the refractive index $1<n \leq 1.0185$, we assume $n(w) \simeq n$ and take an average value of $n$ within the above range $(n=$ 1.006). Assuming the plasma frequency to be much smaller than the incoming neutrino energy, $w_{p}<<E_{1}$ we obtain

$$
S \simeq \frac{T}{8 \pi^{2} \alpha} G_{F}^{2} C_{V}^{2} P_{l}^{2}(n-1)^{3} E_{1}^{2}
$$

Thus the energy intensity of the emitted longitudinal photon by neutrino is proportional to the square of the incoming neutrino energy.

\section{Supernova shock revival}

Observation of neutrino events from supernova SN1987A confirms that neutrino emission is an efficient process of cooling, hot, dense and collapsed stars. The production and propagation of neutrinos are greatly influenced by the collective effects of the stellar plasma. Adams, Ruderman and Woo[11] pointed out that 
plasmon decaying into neutrino-antineutrino pair $(\gamma \rightarrow \nu \bar{\nu})$ would play an important role in the stellar cooling process. Neutrinos are also responsible for the delayed explosion of Type-II supernova. Recent numerical calculations in more than one dimension shows that material behind the stalled shock wave of the supernova can be heated efficiently by neutrinos coming from the neutrinosphere and eventually expel the outer mantle causing the supernova explosion[12, 13, 14]. The neutrino properties get modified when propagate through the plasma medium because of the weak interaction with the background particles. Thus plasma process is of great importance in studying the astrophysical problems. Here we consider the energy deposited by the Cerenkov process described above in the stalled shock wave of the supernova and compare with the Bethe-Wilson mechanism of the shock revival[15].

After the gravitational collapse of a massive star into neutron star, a shock wave is formed and after traveling some distance (about $400 \mathrm{Km}$ [9]) get stalled because most of the kinetic energy in the shock wave is used to dissociate the nuclei. Bethe and Wilson in 1982 showed[15] that, neutrinos from the hot inner core of the supernova are captured by the matter behind the shock through the process $\nu_{e}+n \rightarrow p+e^{-}$and $\bar{\nu}_{e}+p \rightarrow n+e^{+}$and deliver their energy. About $0.1 \%$ of the total energy is sufficient to reheat the matter and eject the stalled shock. In $\mathrm{BW}$ mechanism rate of energy absorbed by a gram of matter at a distance $\mathrm{R}$ is

$$
\dot{E}_{B W}=3 \times 10^{18} L_{\nu 52}\left(\frac{T_{\nu}^{2}}{R_{7}^{2}}\right) \tilde{Y}_{N} \mathrm{erg} / \mathrm{g} / \mathrm{sec},
$$

where $L_{\nu 52}$ is the neutrino luminosity in units of $10^{52} \mathrm{erg} / \mathrm{sec}, R_{7}$ is the distance from the center in units of $10^{7} \mathrm{~cm}, T_{\nu}=5 \mathrm{MeV}$ is the temperature of the neutrino sphere and $\tilde{Y}_{N} \simeq 1$ is the total mean fraction of the nucleon. Here we neglect the contribution due to electron and positron capture as they are correction to this contribution. The total energy absorbed by the stalled shock wave, which has a thickness $d$ and density $\rho$ is

$$
\dot{E}_{B W}=3 \times 10^{18} L_{\nu 52}\left(\frac{T_{\nu}^{2}}{R_{7}^{2}}\right) \tilde{Y}_{N} \operatorname{erg} / g / \sec \times 4 \pi \rho R^{2} d .
$$


For the neutrino luminosity $L_{\nu_{e}}=4 \times 10^{52} \mathrm{erg} / \mathrm{sec}$ and the stalled shock wave density $\rho \simeq 10^{8} \mathrm{~g} / \mathrm{cm}^{3}[10]$ and $R=400 \mathrm{Km}$, the energy absorbed by the shock wave (assuming $100 \%$ absorption) is

$$
\dot{E}_{B W}=9.4 \times 10^{43} d_{c m} \mathrm{erg} / \mathrm{sec} .
$$

So $0.1 \%$ of this is $9.4 \times 10^{42} d_{c m} \mathrm{erg} / \mathrm{sec}$, where $d_{c m}$ is units of $\mathrm{cm}$.

In the Cerenkov process the total energy emitted by neutrinos (we assume that all the radiated photons is absorbed by the medium) per unit time is

$$
\begin{aligned}
\dot{E}_{C} & =S \times(\text { Neutrino flux }) \\
& =\frac{d}{8 \pi^{2} \alpha} G_{F}^{2} C_{V}^{2} P_{l}^{2}(n-1)^{3} E_{1}^{2} \times\left(\frac{L_{\nu}}{E_{1}}\right) .
\end{aligned}
$$

Here we have replaced $T$ by the thickness of the medium $d$. For average electron neutrino energy $E_{1}=12 \mathrm{MeV}$ we obtain $\dot{E}_{C}=1.8 \times 10^{36} \mathrm{~d}_{\mathrm{cm}} \mathrm{erg} / \mathrm{sec}$. Now comparing both the process $(0.1 \%$ of $\mathrm{BW})$ we have

$$
\frac{\dot{E}_{C}}{\dot{E}_{B W}} \simeq 1.9 \times 10^{-7} \text {. }
$$

This shows that the energy deposition by Cerenkov process is extremely small. Both the BW process and the Cerenkov process are weak processes, so in principle the energy deposition in both the processes should not differ too much, and even if there is difference, it should be compensated by the electromagnetic coupling of the neutrino in the medium. But as the Cerenkov process is kinematically allowed only for a very small range of the refractive index $n$ very close to unity and the energy emitted is proportional to $(n-1)^{3}$; for refractive index $n \simeq 1.006,(n-1)^{3}$ is of order $10^{-7}$ thus reduces the contribution for Cerenkov process.

It is a great pleasure to thank Dr. S. Mohanty for many useful discussions. 


\section{References}

[1] V. N. Oraevsky and V. B. Semikoz, Physica A142, 135 (1987); A. Grau and J. A. Grifols, Phys. Lett. B166, 233 (1986); J. C. D’Olive, J. F. Nieves and P. B. Pal, Phys. Rev. D40, 3679 (1989).

[2] J. C. D’Olive, J. F. Nieves and P. B. Pal, Phy. Lett. B365, 178 (1996).

[3] T. Altherr and S. Salati, Nucl. Phys. B421, 662 (1994).

[4] W. Grimus and H. Neufeld, Phys. Lett. B315, 129 (1993).

[5] A. Suzuki et. al, Phys. Rev. D43, 3557 (1991).

[6] A. N. Ioannisian and G. G. Raffelt, hep-ph/9612285.

[7] D. Notzold and G. Raffelt, Nucl. Phys. B307, 924 (1988); P. B. Pal and T. N. Pham, Phys. Rev. D40, 259 (1989).

[8] E. Bratten and D. Segal, Phys. Rev. D48, 1478 (1993).

[9] G. M. Fuller et. al, Astrophys. J. 389, 517 (1992).

[10] S. E. Woosley and T. A. Weaver, Ann. Rev. Astron. Astrophys. 24, 205 (1986).

[11] J. B. Adams, M. A. Ruderman and C. H. Woo, Phys. Rev. 129, 1383 (1963);

C. L. Inman and M. A. Ruderman, Phys. Rev. 140, 1025 (1965).

[12] W. D. Arnet et. al, Ann. Rev. Astron. Astrophys 27, 629, (1989); M. Herant et. al, Astrophys. J., 435, 339 (1994).

[13] A. Burrows, J. Goshy, Astrophys. J. Lett. 416, L75 (1993); H. T. Janka, E. Muller, Astrophys. J., 448, L109 (1995); phys. Rep., 256, 135 (1995); Astron. Astrophys. 306, 167 (1996); A. Burrows, J. Hayes, B. A. Fryxell, Astrophys. J., 450, 830 (1995). 
[14] E. Kh. Akhmedov et. al, hep-ph/9603443.

[15] H. A. Bethe and J. R. Wilson, Astrophys. J. 295, 14 (1985). 
This figure "fig1-1.png" is available in "png" format from: http://arxiv.org/ps/hep-ph/9612375v1 
This figure "fig1-2.png" is available in "png" format from: http://arxiv.org/ps/hep-ph/9612375v1 